\title{
Properties of charge density waves in $\mathrm{La}_{2-x} \mathrm{Ba}_{x} \mathrm{CuO}_{4}$
}

\author{
Young-June Kim, , , G. D. Gu, ${ }^{2}$ T. Gog, ${ }^{3}$ and D. Casa ${ }^{3}$ \\ ${ }^{1}$ Department of Physics, University of Toronto, Toronto, Ontario M5S 1A7, Canada \\ ${ }^{2}$ Department of Condensed Matter Physics and Materials Science, \\ Brookhaven National Laboratory, Upton, New York, 11973-5000 \\ ${ }^{3}$ CMC-CAT, Advanced Photon Source, Argonne National Laboratory, Argonne, Illinois 60439
}

(Dated: November 8, 2018)

\begin{abstract}
We report a comprehensive x-ray scattering study of charge density wave (stripe) ordering in $\mathrm{La}_{2-\mathrm{x}} \mathrm{Ba}_{\mathrm{x}} \mathrm{CuO}_{4}(\mathrm{x} \approx 1 / 8)$, for which the superconducting $T_{c}$ is greatly suppressed. Strong superlattice reflections corresponding to static ordering of charge stripes were observed in this sample. The structural modulation at the lowest temperature was deduced based on the intensity of over 70 unique superlattice positions surveyed. We found that the charge order in this sample is described with one-dimensional charge density waves, which have incommensurate wave-vectors $(0.23,0,0.5)$ and $(0,0.23,0.5)$ respectively on neighboring $\mathrm{CuO}_{2}$ planes. The structural modulation due to the charge density wave order is simply sinusoidal, and no higher harmonics were observed. Just below the structural transition temperature, short-range charge density wave correlation appears, which develops into a large scale charge ordering around $40 \mathrm{~K}$, close to the spin density wave ordering temperature. However, this charge ordering fails to grow into a true long range order, and its correlation length saturates at $\sim 230 \AA$, and slightly decreases below about $15 \mathrm{~K}$, which may be due to the onset of two-dimensional superconductivity.

PACS numbers: 61.05.cf, 74.72.Dn, 71.45.Lr
\end{abstract}

\section{INTRODUCTION}

Over twenty years have passed since the discovery of superconductivity in $\mathrm{La}_{2-\mathrm{x}} \mathrm{Ba}_{\mathrm{x}} \mathrm{CuO}_{4}$ (LBCO) by Bednorz and Muller in $1986 . \frac{1}{*}$ Although the theory of high temperature superconductivity still remains elusive, much understanding of this exciting condensed matter system, and also physics of correlated electron systems in general, have been gained in the past two decades. There have been many important experimental discoveries and theoretical ideas to describe the observed physical properties, and a number of recent review articles cover many of these developments $2,3,4,5$ One of these is the so-called charge and spin stripe picture. That is, doped holes in the cuprates tend to aggregate in one-dimensional domain walls separating regions of antiferromagnetically ordered spin domains. As early as 1988, incommensurate spin fluctuations in cuprate superconductors were observed using neutron scattering. $. .7,8$ These observations stimulated various theoretical work dealing with charged domain walls in two dimensional Hubbard model. 10,11 In 1995, motivated by the theoretical model put forward by Emery and Kivelson, $, 12,13$ Tranquada and coworkers discovered static ordering of charge and spins in $\mathrm{La}_{1.6} \mathrm{Nd}_{0.4} \mathrm{Sr}_{0.125} \mathrm{CuO}_{4}$ (LNSCO) that is consistent with the stripe model $\underline{14}$ The next breakthrough was the discovery of static ordering of spin stripes in $\mathrm{La}_{2-\mathrm{x}} \mathrm{Sr}_{\mathrm{x}} \mathrm{CuO}_{4+\mathrm{y}}$ (LSCO) over a wide doping range: $\frac{15,16,17}{1 n}$ addition to these static incommensurate ordering of spins in LSCO, incommensurate spin fluctuations have been observed in a number of materials, including $\mathrm{YBa}_{2} \mathrm{Cu}_{3} \mathrm{O}_{6+\mathrm{x}}(\mathrm{YBCO}) \underline{18}$

Despite such extensive experimental work on the incommensurate spin fluctuations and ordering in cuprate superconductors, experimental studies on the charge counterpart have been relatively scarce. In recent years, the scanning tunneling spectroscopy (STS) technique has attracted much attention due to its ability to provide real space image of charge distribution on $\mathrm{Bi}_{2} \mathrm{Sr}_{2} \mathrm{CaCu}_{2} \mathrm{O}_{8+\delta}$ and $(\mathrm{Ca}, \mathrm{Na})_{2} \mathrm{CuO}_{2} \mathrm{Cl}_{2}$, etc. Although these STS studies provide unprecedented information on the inhomogeneous distribution of charge density and superconducting gap $\frac{19,20,21,22}{1}$ due to the surface sensitive nature of the technique, its application has been so far limited to a subset of cuprate samples. In contrast, neutron scattering investigation of incommensurate correlation has been observed in many different materials. Although there have been recent neutron scattering studies on YBCO, the bulk of studies on stripes have been carried out with LSCO and LNSCO 14,15,16,23 Recently, it has become possible to synthesize high quality large single crystals of LBCO, and various experimental studies have been carried out to elucidate the charge and spin properties of LBCO $.24,25,26,27,28,29,30$

However, neutron scattering investigation of the charge counterpart of the stripe order has been very limited, due to its indirect coupling to charge degrees of freedom, and also its low signal to noise ratio. On the other hand, x-ray scattering couples directly to the charge degree of freedom, and is still a bulk probe (5-10 microns). Except for when the incident photon energy is near the absorption edges, the largest contribution to the x-ray scattering intensity comes from the structural modulations accompanying charge order. In this sense, x-ray scattering is similar to neutron scattering, but one can use a synchrotron source and obtain very high intensity and high momentum resolution. The first x-ray study of the charge stripes was done with very high en- 
ergy x-rays $(\mathrm{E}=100 \mathrm{keV})$ at HASYLAB by Zimmermann and coworkers 31 They were able to elucidate the stacking structure and temperature dependence of the charge stripe ordering in their study of LNSCO. Further studies of LNSCO $\mathrm{x}=0.15$ sample have been carried out at the same facility ${ }^{32.33}$ In addition, the structural phase transition and its relationship with the charge ordering has been investigated with regular (low energy) x-ray scattering on $\mathrm{La}_{1.875} \mathrm{Ba}_{0.125-\mathrm{x}} \mathrm{Sr}_{\mathrm{x}} \mathrm{CuO}_{4}$ (LBSCO) $\stackrel{34}{\text { Recent }}$ availability of the $\mathrm{LBCO}$ crystals have made it possible to carry out more detailed investigation of LBCO using soft $\mathrm{x}$-ray resonant scattering. 25

In this paper, we report our comprehensive investigation of charge stripe ordering in LBCO using synchrotron $\mathrm{x}$-ray scattering. As previously reported, clear peaks corresponding to the charge stripe ordering were observed. We confirmed some of the earlier observations for LNSCO in the current LBCO sample, which indicates that the charge stripe ordering in these two compounds are very similar. In addition, we were able to study the structure of the charge ordering in detail by surveying the intensity of various charge stripe superlattice peaks. We find that the charge stripe order is described with a sinusoidal charge density wave with incommensurate wave-vector $(0.23,0,0.5)$ in this sample, and no higher harmonics were observed. Short-range charge stripe correlation sets in as soon as the structural transition occurs in this sample and the stripe correlation grows into a large scale charge ordering around $40 \mathrm{~K}$, which is close to the spin density wave ordering temperature. However, this charge ordering fails to grow into a true long range order, and its correlation length saturates at $230 \AA$, and slightly decreases below about $15 \mathrm{~K}$, which may be due to the onset of two-dimensional (2D) superconductivity as suggested by $\mathrm{Li}$ et al ${ }^{29}$

After first describing the experimental details in the next section, we will present our results on the structural phase transition, incommensurability, charge order structure, and temperature dependence in order. Some of the implications of these experimental results will be discussed in Section VI.

\section{EXPERIMENTAL DETAILS}

Preliminary x-ray scattering experiments were carried out at the X22C beamline at National Synchrotron Light Source (NSLS). Additional high-resolution data were obtained at the 9ID (CMC-CAT) beamline at Advanced Photon Source (APS). In both experiments, the incoming photon was monochromatized by a double bounce $\mathrm{Si}(111)$ monochromator in vertical scattering geometry. Either graphite (002) or Si (111) reflections were used to reduce the background and also to improve the momentum resolution. Data were obtained both in the (HK0) and the (H0L) scattering plane with either 8.9 $\mathrm{keV}$ (NSLS) or $12 \mathrm{keV}$ (APS) photons. The samples used in our measurements were grown with traveling-solvent floating zone technique. A small piece from a large boul of single crystals was cut to reveal the [0KL] surface, which is polished. In the temperature range studied, the LBCO sample is in its low-temperature tetragonal (LTT) phase. However, for consistency, we will use the notation following the high-temperature tetragonal (HTT) lattice parameters, with $a=3.787 \AA$ and $c=13.24 \AA$.

The superconducting transition temperature of the sample was determined by measuring diamagnetic signal with Quantum Design MPMS SQUID magnetometer, as shown in the Fig. 1]inset. The onset temperature is about $\sim 6 \mathrm{~K}$. We have not carried out further characterization to determine the doping level accurately, but from the suppression of $T_{c}$, we can estimate that the doping is not very different from $\mathrm{x}=0.125$. All our $\mathrm{x}$-ray measurements were carried out on a single piece of crystal, and the magnetization was measured with a small piece cut from this original piece.

\section{LOW TEMPERATURE TETRAGONAL STRUCTURE}

One of the most important aspects of LBCO (and also LNSCO) that distinguishes this from other cuprate superconductors is its low temperature structure. Specifically, LBCO has the so-called LTT structure, which can stabilize static ordering of charge stripes. It is well-known that the crystal structure of LSCO at high temperature is tetragonal with flat $\mathrm{CuO}_{2}$ planes (Symmetry $I 4 / \mathrm{mmm}$ ). As temperature is lowered, structural phase transition to an orthorhombic phase called low-temperature orthorhombic (LTO, symmetry group Bmab) occurs, in which the $\mathrm{CuO}_{2}$ plane is buckled, such that the $\mathrm{Cu}-\mathrm{O}-$ $\mathrm{Cu}$ bond angle deviates from 180 degrees 35 One should note that the buckling is caused by rotation of $\mathrm{CuO}_{6}$ octahedra along the axis making 45 degrees with the $\mathrm{Cu}$ O-Cu direction. (See Fig. 2(a)) This is the low temperature structure of doped LSCO samples as well, at least up to $\mathrm{x}=0.2$. For $x>0.2$, the low temperature structure remains tetragonal. LBCO also goes through the same structural phase transition from high temperature tetragonal (HTT) phase to low temperature orthorhombic (LTO) phase at $\sim 200 \mathrm{~K}, \underline{36,37}$

In addition to this HTT-LTO transition, LBCO goes through another structural phase transition around $55 \mathrm{~K}$, and the low temperature phase of LBCO again has tetragonal structure. However, this LTT structure (symmetry group $P 4_{2} / \mathrm{ncm}$ ) is different from the HTT phase, and the $\mathrm{CuO}_{2}$ plane remains buckled. The tetragonal symmetry (with larger unit cell) is achieved by additional tilting of the $\mathrm{CuO}_{6}$ octahedra. This time, the rotation axis is along the $\mathrm{Cu}-\mathrm{O}-\mathrm{Cu}$ bond direction [See Fig. 2(a)]. As a result of this rotation, stripes running along the $\mathrm{Cu}-$ $\mathrm{O}-\mathrm{Cu}$ bond direction can be pinned to the lattice. This is believed to be the origin of static ordering of charge and spin stripes in LBCO and LNSCO. In Fig. 11 the temperature dependence of the $\left(\begin{array}{lll}5 & 0 & 0\end{array}\right)$ superstructure peak is 


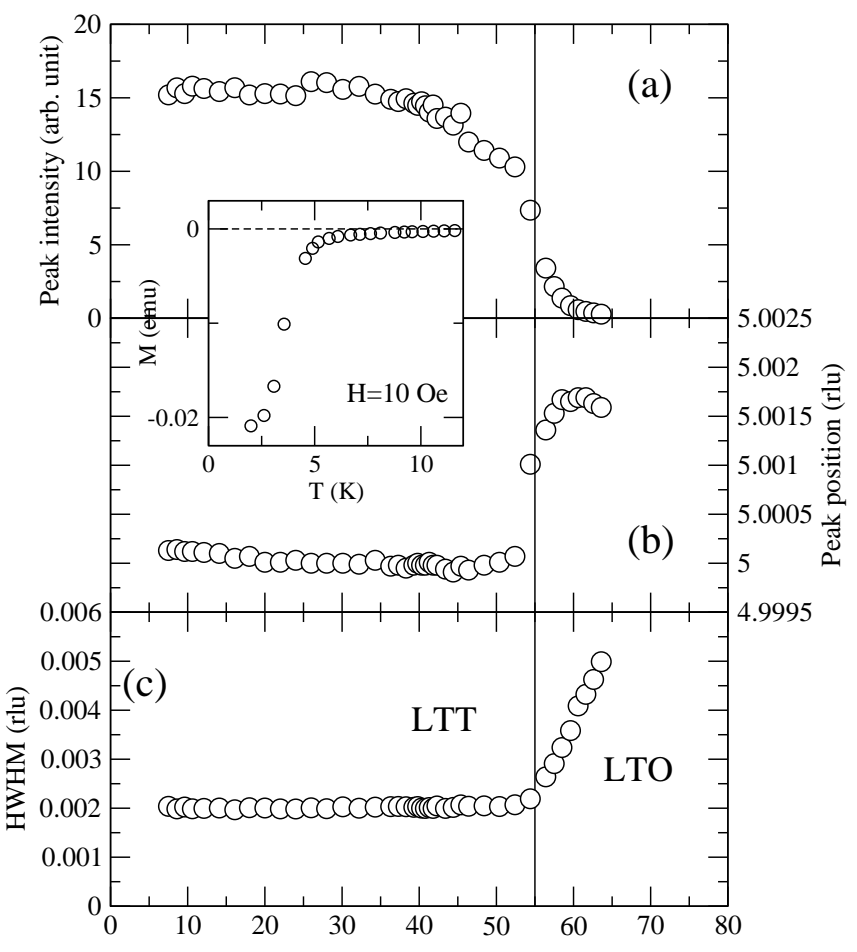

FIG. 1: The temperature dependence of the (a) peak intensity, (b) nominal peak position, and (c) peak width of the (5 0 0) LTT superstructure peak. The vertical line corresponds to the LTT-LTO structural phase transition temperature $T_{s}$. Inset: Temperature dependence of magnetization showing superconducting transition.

presented. The peak intensity drops abruptly as temperature is raised above $T_{s}=55 \mathrm{~K}$, while the nominal peak position suddenly jumps to higher value at the same temperature, both signaling discontinuous structural phase transition. However, the peak width is resolution limited at $T<T_{s}$, and grows continuously for $T>T_{s}$. Note that this peak is resolution limited in all directions at low temperatures even with the best resolution setup, indicating that the LTT phase is established over $1400 \AA$.

Earlier neutron and electron diffraction studies on poly-crystalline samples have reported that the LTT phase is short-range ordered, and the LTO phase persists even at the lowest temperature studied, 36,38 while the neutron diffraction study by Katano and coworkers found that LTT fraction is much larger than LTO fraction. $\underline{37}$ Our results seem to be consistent with the latter result. That is, the LTT phase is developed fully at low temperatures, over a macroscopic region. The temperature dependence of the correlation length suggests that the LTT phase nucleates at temperatures above $T_{s}$, and grows in size until it reaches macroscopic scale at temperatures between $50 \mathrm{~K}$ and $60 \mathrm{~K}$. The salient point here is that the LTT phase is fully developed by the time the temperature reaches the charge ordering temperature, and that the LTT order is long-ranged, while the charge order is (a)

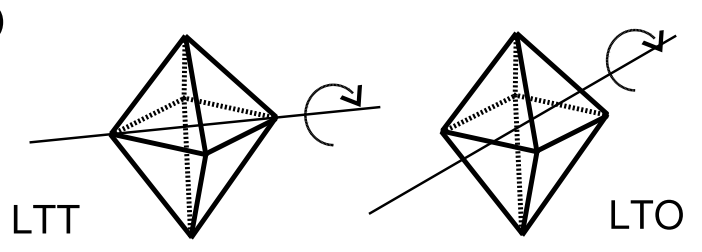

(b)

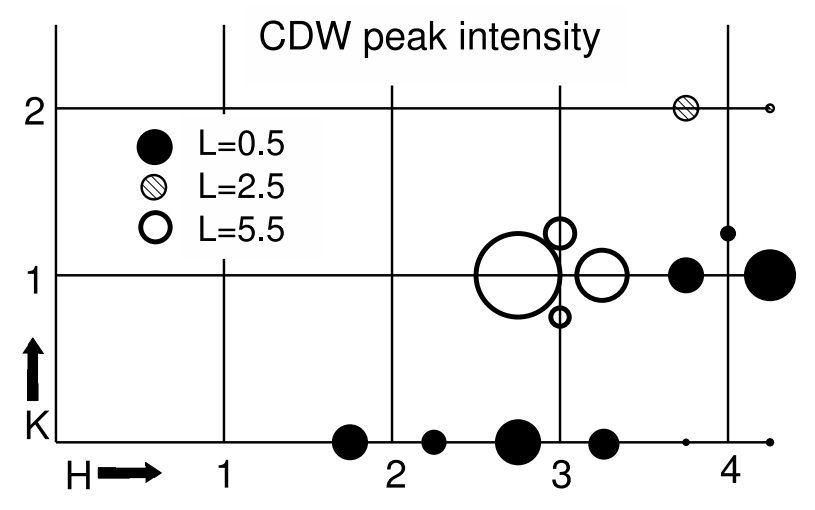

FIG. 2: (a) Schematic representation of the rotation of $\mathrm{CuO}_{6}$ octahedra in the LTT phase (left) and the LTO phase. (b) Reciprocal space diagram to show the intensity distribution of the charge order peaks. The location and the radii of the circle correspond to the position and intensity of the observed peak. Note that satellite peaks along the $K$ direction are only observed around the Bragg peaks with non-zero $K$ values, as described in the text.

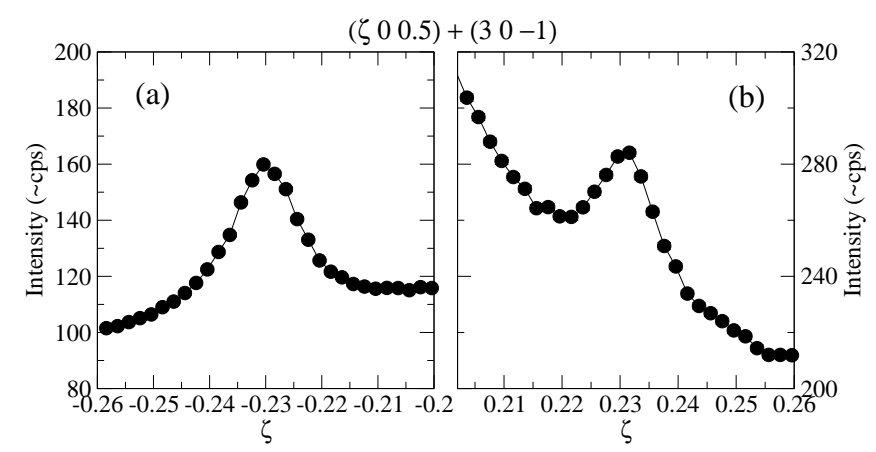

FIG. 3: Representative scans through the CDW peaks near the $\mathbf{G}=(3,0, \overline{1})$. Scans along the $\mathrm{H}$ direction on either side of the Bragg peak are shown.

short-ranged as we will show later.

\section{GROUND STATE}

We were able to survey about 70 unique reciprocal lattice positions where charge order peak is expected to ex- 
TABLE I: Summary of the experimentally observed CDW peaks at $\mathbf{G}+(\epsilon, \mathbf{0}, \mathbf{0 . 5})$ and SDW peaks at $(0.5+\delta, 0.5+\delta, 0)$ for a number of cuprates.

\begin{tabular}{|c|c|c|c|c|c|}
\hline Sample & doping $(\mathrm{x})$ & $\epsilon \mathrm{CDW}$ & $\delta \mathrm{SDW}$ & Note & Reference \\
\hline LNSCO & 0.12 & 0.236 & 0.118 & neutron & 14 \\
\hline LNSCO & 0.12 & 0.236 & & x-ray & 31 \\
\hline LNSCO & 0.15 & 0.256 & & x-ray & 32 \\
\hline LNSCO & 0.15 & 0.258 & 0.134 & $\mathrm{x}$-ray+neutron & 33 \\
\hline LBCO & 0.125 & 0.236 & 0.118 & neutron & 39 \\
\hline $\mathrm{LBCO}$ & 0.125 & 0.245 & & rsxs & 25 \\
\hline LBSCO & 0.125 & 0.24 & & x-ray & 34 \\
\hline LBCO & 0.12 & 0.230 & & x-ray & This work \\
\hline
\end{tabular}

ist. These are $\mathbf{G} \pm \mathbf{g}_{\mathbf{1}}$ and $\mathbf{G} \pm \mathbf{g}_{\mathbf{2}}$, where $\mathbf{G}$ is a Bragg peak position, and $\mathbf{g}_{1}=(\epsilon, 0,0.5)$ and $\mathbf{g}_{2}=(0, \epsilon, 0.5)$ are incommensurate charge order wavevectors. Some of these peak intensities are reported in Fig. 2(b), in which the size of a circle is proportional to the peak intensity at this position. These measurements were carried out around $T=10 \mathrm{~K}$, which is above the superconducting transition temperature. In this section we present this survey result along with its implication for the ground state structure of the charge stripe order in LBCO.

\section{A. Incommensurate charge density wave}

In Fig. 3, we show representative scans through the charge order peak obtained around $\mathbf{G}=(3,0, \overline{1})$. Scans along the longitudinal direction $(\mathrm{H})$ on either side of the Bragg peak are shown in Fig. 3(a) and (b), respectively. It is clear that the charge order peak is located at the incommensurate wavevector, $\mathbf{g}_{1}=(\epsilon, 0,0.5)$, with $\epsilon=0.230(2)$. That is, the periodicity of the charge order is not exactly 4 times the lattice constant, and it is indeed incommensurate with the lattice. Therefore, the "charge stripe order" in LBCO is better described as an incommensurate charge density wave (CDW). The value of $\epsilon$ is consistently around $\sim 0.230$ for all the peaks studied in our experiments. This incommensurability shows very little temperature dependence over the whole range studied in our experiments. This is also consistent with the neutron scattering results of Fujita et al., in which they observed $\epsilon=0.236$ for the LBCO sample with $T_{c} \approx 4 \mathrm{~K}$.

In the conventional stripe picture for $\mathrm{x}=1 / 8$, one can construct a charge order model with exactly $4 a$ periodicity due to the commensurate nature of the doping. That is, if each stripe carries charge of half an electron as shown in recent x-ray study, ${ }^{25}$ then the charge order unit cell is enlarged by fourfold, and CDW peak at the incommensurability of $\epsilon=0.25$ should be observed. In Table I. experimental values for the CDW incommensurability in a number of samples are summarized. Although the CDW and SDW incommensurability are clearly correlated $(\epsilon=2 \delta)$, no obvious correlation between the doping $(\mathrm{x})$ and $\epsilon$ is found, and none of the data shows exactly $\epsilon=0.25$. Empirically, $\epsilon<2 x$ is observed in all the stud- ies. Our observation of $\epsilon=0.230$ therefore is consistent with previous measurements. One explanation for this empirical observation may come from doping dependence of the incommensurability. It is well known that SDW incommensurability in LSCO samples follow $\delta=x$ in the underdoped regime, ${ }^{23}$ and if one assumes that $\epsilon=2 x$ should hold in the CDW order, our sample may have $x=0.115$ rather than nominal $x=0.125$. The superconducting $T_{c}$ of LBCO samples are rapidly suppressed near the $x=1 / 8$ doping, and $T_{c}$ can be a good measure of actual doping level of the sample. From the phase diagram of LBCO,, 36 the $x=0.115$ sample should have $T_{c}$ between 5 and $10 \mathrm{~K}$, which is consistent with the measured value.

In Fig. 4, we show the $\mathrm{Q}$ dependence of the scattering intensity at ( $\mathrm{H} \quad 0 \quad 5.5)$ between $\mathrm{H}=4.2$ and 4.8 . In order to display the scattering intensity due to the CDW at $T=10 \mathrm{~K}$, we have subtracted the temperature independent background intensity at $T=52 \mathrm{~K}$. Note that the vertical axis is shown in logarithmic scale to magnify any small intensity. Considering the background noise, we should be able to detect any higher harmonic peaks if they were larger than $1 \%$ of the first harmonic. This observation strongly suggests that the structural modulation due to CDW can be well described by a simple sinusoidal modulation with doping-dependent incommensurate wavevector $(0.23,0,0.5)$.

\section{B. Structural modulation}

In Fig. 2(b), we show intensity distribution of observed superlattice peaks in the two-dimensional plane of $(\mathrm{H}$ $\mathrm{K}$ ) for a number of $\mathrm{L}$ values. One should notice that while the CDW peaks of $(H, 0,0) \pm \mathbf{g}_{1}$ are observed, $(H, 0,0) \pm \mathbf{g}_{2}$ CDW peaks are not observed. That is, only a pair of superlattice peaks are observed around the $(H, 0,0)$ Bragg peak. When there is a finite $K$ component, small $\mathbf{g}_{2}$ type CDW peaks could be observed: e.g., $(3,1 \pm \epsilon, 5.5)$ and $(4,1+\epsilon, 2.5)$. The superlattice peak intensity arising from a structural modulation vector, $\mathbf{u}_{r}$, is generally proportional to $\left(\mathbf{Q} \cdot \mathbf{u}_{r}\right)^{2}$. Since $\mathbf{Q}$ is along the $\mathbf{a}^{*}$ direction for $\mathbf{G}=(H, 0,0), \mathbf{g}_{2}$-type superlattice peaks are suppressed. Robertson and coworkers recently proposed that one of the experimental signatures of $1 \mathrm{D}$ stripe 


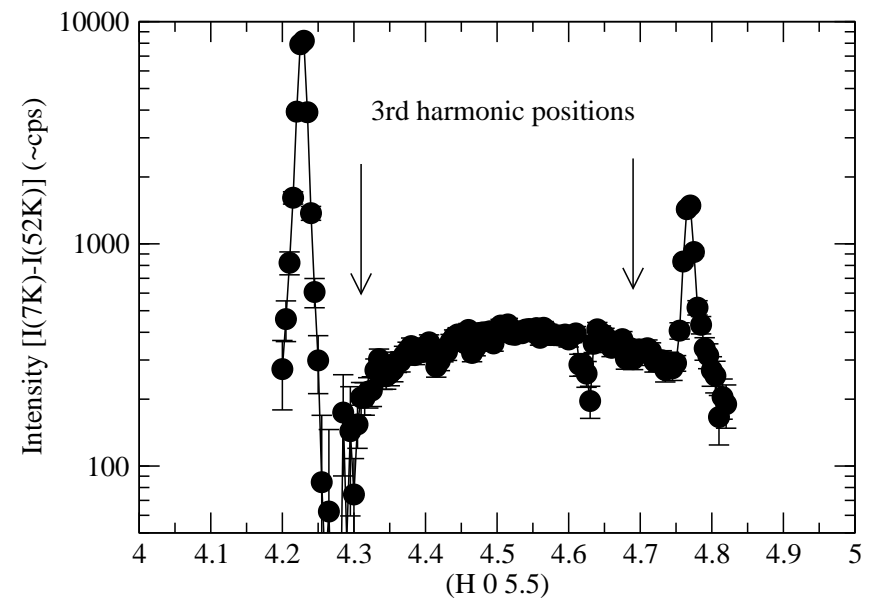

FIG. 4: A scan along the ( $\mathrm{H} 0$ 5.5) direction. In order to show the intensity due to the CDW order, intensity difference between the same scan obtained at two different temperatures $(7 \mathrm{~K}$ and $52 \mathrm{~K})$ is plotted. Two strong peaks correspond to the main CDW peaks at $( \pm \epsilon, 0,0.5)$. Logarithmic scale is used to emphasize the lack of any higher harmonic peaks. The arrows denote expected positions for third harmonic peaks: $(5,0,7)-3 \mathbf{g}_{1}$ and $(4,0,4)+3 \mathbf{g}_{1}$.

charge ordering is 4 superlattice peaks, while 8 superlattice peaks are expected for a checkerboard type charge order $\stackrel{40}{\underline{4}}$ We have also searched for additional superlattice peaks along the diagonal direction $\mathbf{q}=( \pm \eta, \pm \eta, l)$ around $\mathbf{G}=(4,2,6)$ at low temperatures without success. Therefore, our result seems to indicate 1D nature of stripes.

The stacking structure of these 1D stripes was proposed by Tranquada and coworkers, and confirmed in the x-ray study by Zimmermann and coworkers. Specifically, if the $\mathrm{CuO}_{6}$ octahedra in one copper oxygen layer $(\mathrm{z}=0)$ is tilted along the a-direction, the octahedra in the neighboring layer $(\mathrm{z}=0.5)$ is tilted along the b-direction. This naturally stabilizes the stripes running along the $\mathrm{b}$-direction and the a-direction for $\mathrm{z}=0$ and $\mathrm{z}=0.5$, respectively. Therefore, normally one would expect to observe four satellite reflections, were it not for the $\left(\mathbf{Q} \cdot \mathbf{u}_{r}\right)^{2}$ factor. The Coulomb interaction between charge stripes causes the stripes in the $\mathrm{z}=1$ layer to be located in between the $\mathrm{z}=0$ stripes, resulting in 2 unit cell (4 layer) periodicity along the c-direction. As a result, all the stripe peaks are observed at $L=0.5,1.5, \ldots$.

The intensity modulation along the L-direction is nonmonotonic as shown in Fig. 5 for a number of fixed inplane wavevectors. This observation is somewhat puzzling, since one expects the above mentioned $\left(\mathbf{Q} \cdot \mathbf{u}_{r}\right)^{2}$ dependence. The main reason for the non-trivial Ldependence of the intensity modulation is the position of La and apical oxygens due to the tilting of octahedra in the LTT phase. Specifically, in LTT phase, the La atoms are slightly displaced from its original position in the LTO phase, so that they no longer are lo-

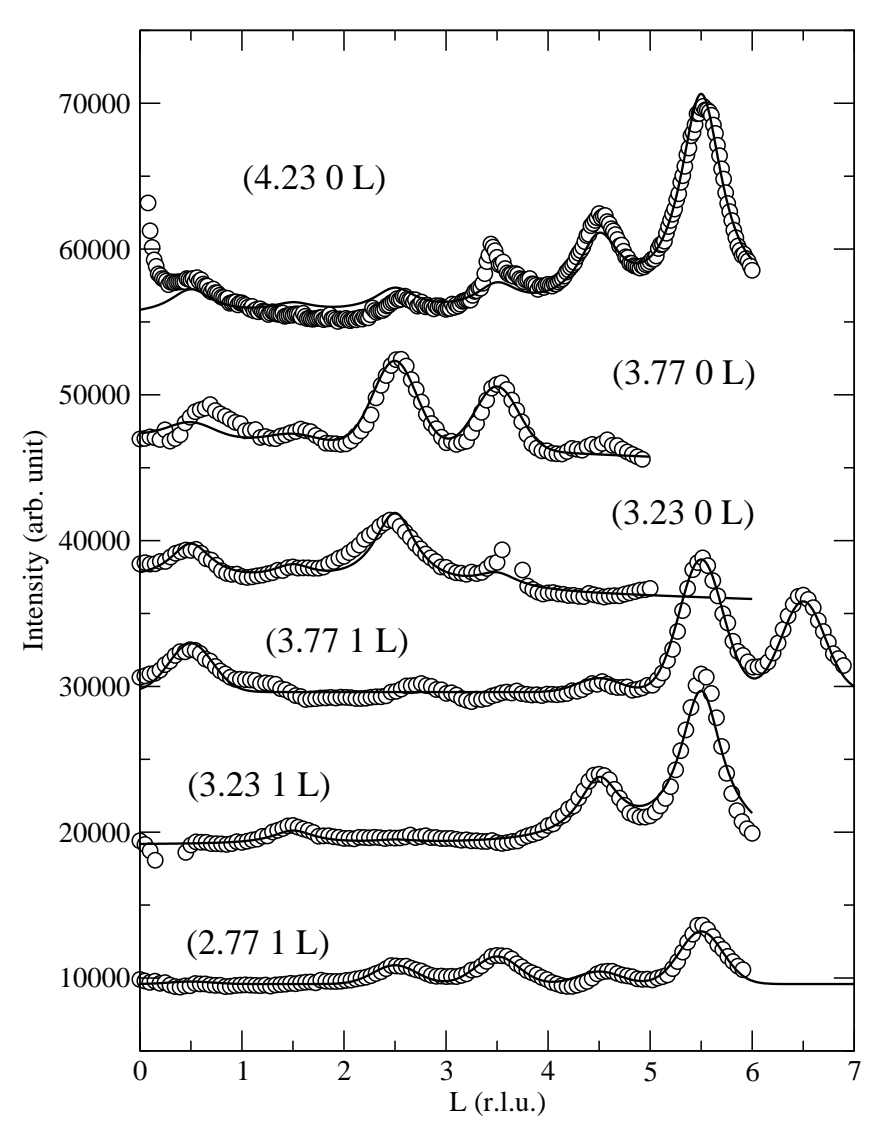

FIG. 5: L-dependence of the CDW peaks. The in-plane positions are labeled. The difference between the scans taken at $7 \mathrm{~K}$ and $55 \mathrm{~K}$ are plotted, and each scan is shifted for clarity.

cated on top of the $\mathrm{Cu}$ atom. Instead, the La atoms are found at $\left( \pm \delta, 0, \pm z_{L a}\right)$ and $\left(\frac{1}{2}, \frac{1}{2} \mp \delta, \frac{1}{2} \mp z_{L a}\right)$, with $\delta=0.0106$ and $z_{L a}=0.36 \frac{37}{4}$ As a result the structure factor of the CDW peaks acquire $\Delta z_{L a} \sin 2 \pi\left(z_{L a} L-\delta h\right)$ and $\Delta z_{L a} \sin 2 \pi\left(z_{L a} L-\delta k\right)$ factors, where $\Delta z_{L a}$ is the c-axis modulation amplitude of the La atoms.

In their neutron scattering study of charge ordering in $\mathrm{La}_{2} \mathrm{NiO}_{4.125}$, Tranquada et al. used a simple incommensurate modulation model to fit the observed superlattice peak intensities, and elucidate atomic displacements in the modulated structure $\stackrel{\underline{41}}{=}$ However, unlike neutron scattering, it is notoriously difficult to obtain absolute $\mathrm{x}$-ray scattering intensity over a wide $\mathbf{Q}$ range, due to the sample absorption and the $\mathbf{Q}$-dependence of the atomic form factor. As a result, although we followed the analysis in Ref. 41 to model the structural modulation due to the charge stripe order in LBCO, we left the overall amplitude as a fitting parameter. Refinement of the structural modulation model is beyond the scope of this work, and will be addressed in a future publication.

We focus here on reproducing the L-dependence of the charge order peak intensity. Following the treatment in Ref. 41, we carried out similar least squares fitting analysis of the observed peak intensities (shown in Fig. 2(b) 

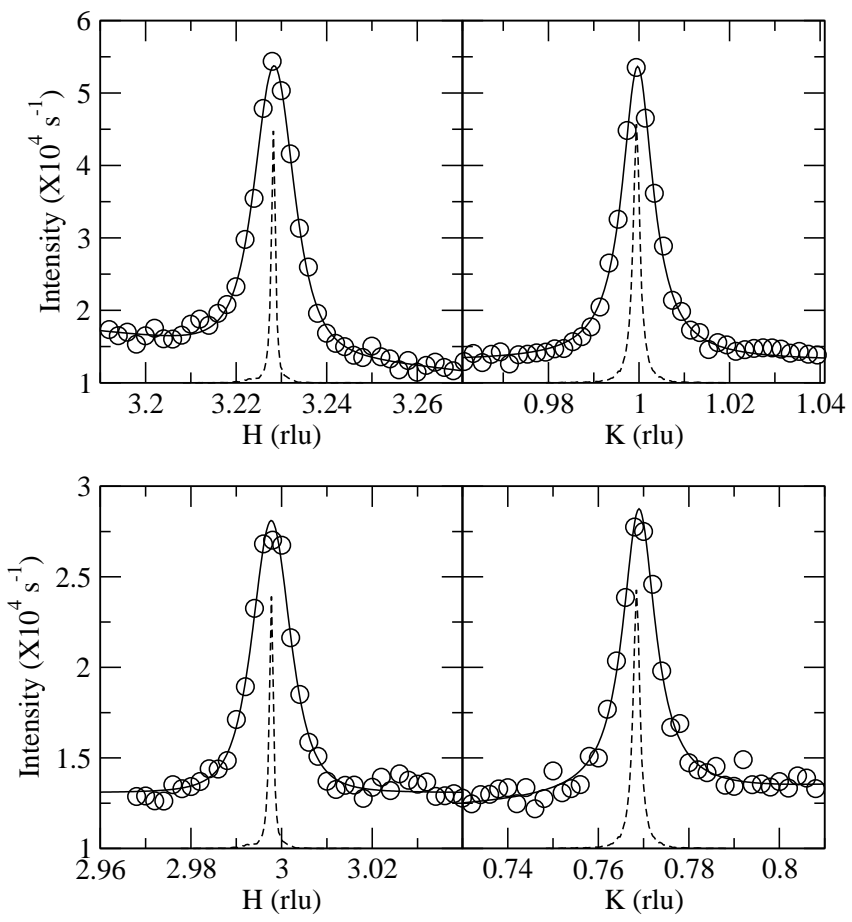

FIG. 6: Representative $\mathrm{H}$ and $\mathrm{K}$ scans around $\mathbf{G}=(\mathbf{3}, \mathbf{1}, \mathbf{5})$ obtained with high-resolution setup at $7 \mathrm{~K}$. The solid lines are fits to $2 \mathrm{D}$ Lorentzian as described in the text, and the dashed lines are instrumental resolution.

and Fig. 5). It turns out that the fitting result is rather insensitive to the modulation of oxygen atoms, as expected from their small x-ray form factor. We also noticed that the in-plane motion of La atoms is negligible, and the most important modulation is the in-plane modulation of copper and out-of-plane (c-direction) modulation of the La atoms. The solid lines shown in Fig. 5 are produced with the least squares fitting results for the intensity of observed CDW peaks. The background and the overall intensity were varied to match the observed data, and the widths of the peaks are fixed at 0.25 r.l.u. As one can see in Fig. 5 that the L-dependent intensity modulation is well described by our analysis.

\section{Correlation length}

In Fig. 6. we show representative scans at two of the positions marked in Fig. 2(b). The solid line is a fit to 2D Lorentzian line shape. One can clearly see that the peak width is much broader than the instrumental resolution (dashed lines) as measured at the nearby Bragg peak position. In addition, one can see that the CDW peaks are quite isotropic in both $\mathrm{H}$ and $\mathrm{K}$ direction. In fact, our fitting results give consistently similar widths along the $\mathrm{H}$ and $\mathrm{K}$ direction in almost all measured peaks. The correlation length, which is obtained as an inverse of the the Lorentzian peak width is about $200-250 \AA$. This in-plane isotropy of the CDW peak may seem puzzling, considering the 1D nature of the CDW order, as shown in previous section. However, this isotropic correlation length can be understood if one considers the alternating stripe directions in neighboring layers. In other words, the stripe ordered region always has both $\mathrm{H}$-stripe and K-stripe component, which makes it isotropic.

We note that the observed correlation length is of similar order of that observed by Abbamonte et al. $\stackrel{25}{=}$ but much longer than those in LNSCO $\stackrel{42}{\Perp}$ presumably reflecting better crystalline quality of the LBCO sample, compared to LNSCO. This is also consistent with the neutron scattering measurements by Fujita and coworkers, $\frac{39}{}$ although the momentum resolution in their study was quite poor. We emphasize however that the charge stripe correlation length is not limited by the structural disorder. As shown in Sec. III, the LTT-LTO structural phase transition is of first order nature, and the LTT superlattice peak is resolution limited at all temperatures.

\section{TEMPERATURE DEPENDENCE}

\section{A. CDW phase transition}

In Fig. 7, we show the temperature dependence of the $(3.23,1,5.5)$ CDW superlattice peak. From top to bottom, H, K, and L scans obtained at different temperatures are shown with different symbols. The horizontal bars in each panel denote the instrumental resolution widths obtained at the $(3,1,4)$ Bragg peak position. Note the different scale used in the $\mathrm{H}, \mathrm{K}$ scans and the $\mathrm{L}$ scan. The scans are quite broad in all three directions as discussed before, and the width does not seem to change very much below $40 \mathrm{~K}$. In addition, we observe that the maximum peak intensity occurs not at the lowest temperatures, but at around $15 \mathrm{~K}$. In order to analyze the temperature dependence in detail, we fitted the $\mathrm{H}$ and $\mathrm{K}$ scans to a 2D Lorentzian lineshape, as shown in Fig. 6. In Fig. 8, we present such fitting results for two Q positions, $(3.23,1,5.5)$ and $(3,0.77,5.5)$, both around the $(316)$ Bragg peak. Note that these peaks have CDW modulation vector $\mathbf{g}_{\mathbf{1}}$ and $\mathbf{g}_{\mathbf{2}}$, respectively. Due to the $\left(\mathbf{Q} \cdot \mathbf{u}_{r}\right)^{2}$ factor, the $(3.23,1,5.5)$ peak is much stronger, so that the intensities plotted in Fig. 8 are scaled to match. On the other hand, the inverse correlation lengths, $\kappa(T)$, for these two peaks match exactly without any adjustable parameter. In Fig. \&(b), only $\kappa_{H}$ and $\kappa_{K}$ of the $(3.23,1$, 5.5) peak are shown.

As expected from earlier studies, the CDW peak intensity gradually decreases and appears to vanish above $\sim 40 \mathrm{~K}$. In addition to the peak intensity, we also plotted the integrated intensity in Fig. 8 (c), which should be proportional to the square of the CDW order parameter. What is striking in this plot is the difference between the behavior of the integrated intensity and the peak intensity. Below about $15 \mathrm{~K}$, the integrated intensity remains constant, while the peak intensity drops as tem- 


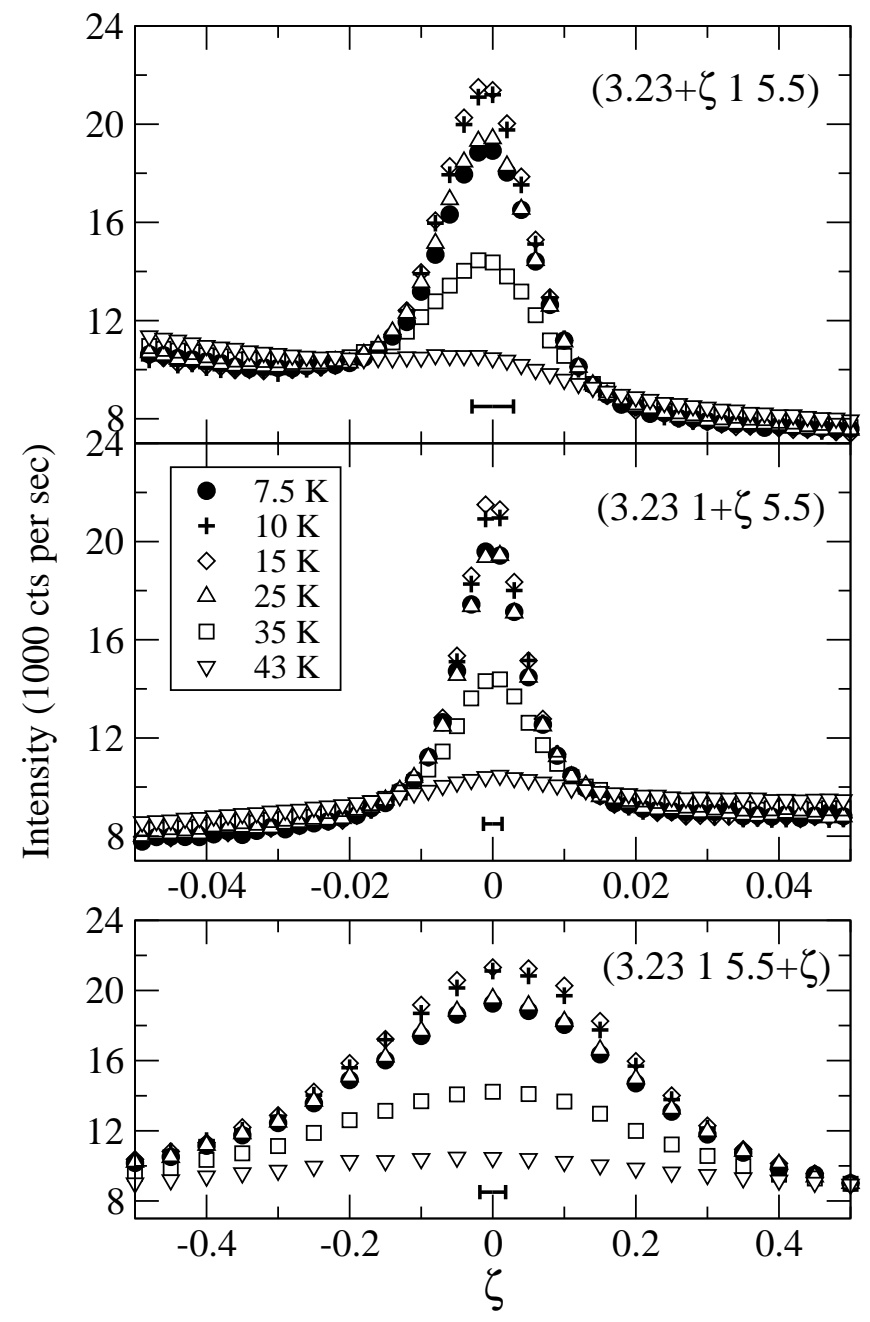

FIG. 7: Scans across the $(3.23,1,5.5)$ CDW peak at different temperatures. From top to bottom, H, K, and L scans are plotted as a function of deviation, $\zeta$, from the peak position. The horizontal bars denote the instrumental resolution.

perature is decreased. While the peak intensity almost goes away above $40 \mathrm{~K}$, the integrated intensity exhibits a cusp around $\sim 42 \mathrm{~K}$, but persists up to the structural transition temperature around $55 \mathrm{~K}$. In order to understand this behavior, one should consider the temperature dependence of the correlation length. Since integrated intensity of a Lorentzian peak is given by peak height multiplied by peak width, the observed decrease in the peak intensity indicates increase in the peak width. This expectation is borne out by the fitting results, which shows small increases in the peak widths below $15 \mathrm{~K}$.

\section{B. Short range charge correlation}

Let us first examine the temperature dependence of correlation lengths above 40 K. In Fig. 9] scans obtained in this high temperature range are plotted. The in-plane

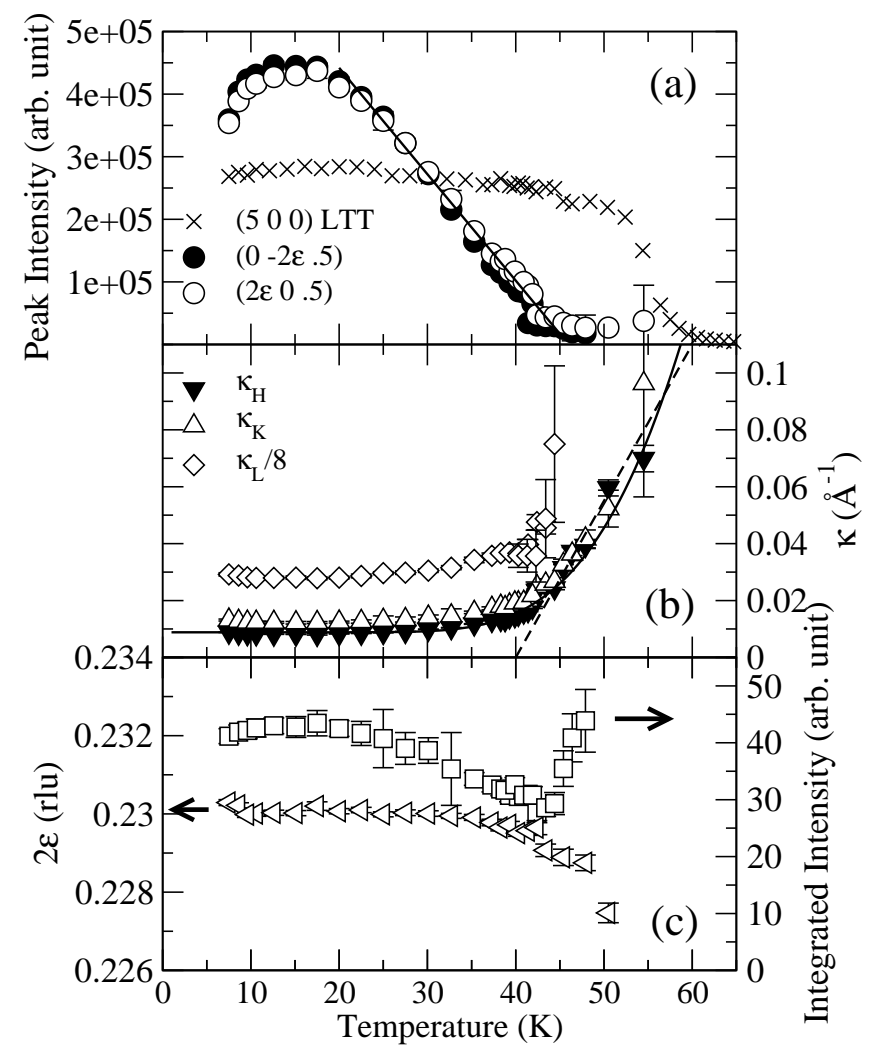

FIG. 8: Temperature dependence of the fitting parameters for the CDW peak intensities around the $(3,1,5)$ position are shown. (a) The open and closed symbols are the peak intensities for the $(3.23,1,5.5)$ and the $(3,0.77,5.5)$ peak, respectively. Also shown in this figure is the temperature dependence of the LTT superlattice peak $(5,0,0)$. (b) The peak widths (inverse correlation length) along the $\mathrm{H}$ and $\mathrm{K}$ directions are shown in closed and open triangles, respectively. These are obtained by scanning the $(3.23,1,5.5)$ peak along the $\mathrm{H}$ and $\mathrm{K}$ directions. The other satellite peak widths exhibit identical temperature dependence. The peak widths along the L-direction are shown in diamond. The solid and dashed lines are fits described in the text. (c) The temperature dependence of the incommensurability $2 \epsilon$ is plotted as triangles, while that of the integrated intensity is plotted with squares. Both quantities were obtained at the $(3.23,1,5.5)$ position.

scans shown in Fig. 9(a) and (b) are the same types of scans as the ones shown in Fig. 7 but at higher temperatures. One can clearly see that the peak located at the CDW order wavevector is still visible at these temperatures, and broadens as temperature increases. That is, the in-plane correlation length contracts significantly, and reaches about $16 \AA$ around $55 \mathrm{~K}$, which is roughly the distance between stripes. Fitting results at these high temperatures are also plotted in Fig. 8, In order to model the temperature dependence of the correlation length, we adopted the phenomenological expression used in Ref. 42 


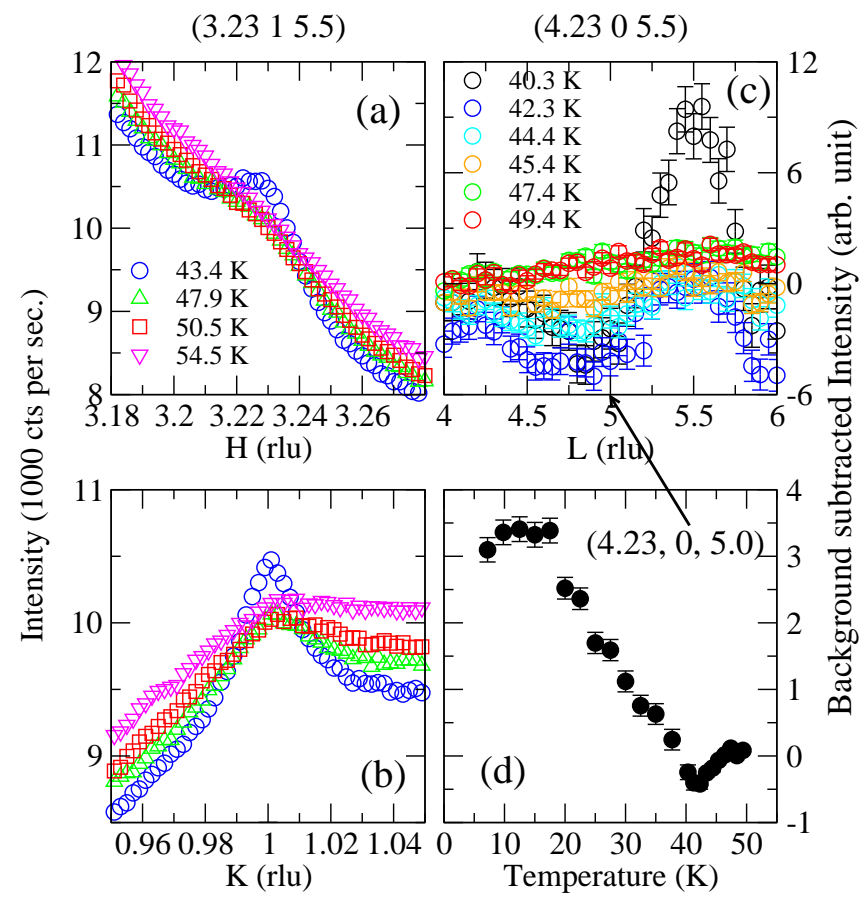

FIG. 9: (Color online) (a)-(c) H, K, and L scans at high temperatures, respectively. The $\mathrm{H}$ and $\mathrm{K}$ scans shown in (a) and (b) are the same as those shown in Fig. 7, except that these are obtained at higher temperatures. (c) Background subtracted intensity around the $(4.23,0,5.5)$ peak is shown. The background scan was taken at $50.4 \mathrm{~K}$, which is justified by the observation that the L-dependence goes away above $\sim 47 \mathrm{~K}$. (d) The background subtracted intensity of $(4.23,0$, $5)$ position as a function of temperature. This position corresponds to the center point shown in part (c) and indicates the intensity of the $2 \mathrm{D}$ fluctuations.

to describe spin stripe inverse correlation length:

$$
\kappa=\kappa_{0}+A e^{-B / k_{B} T}
$$

and obtained $\kappa_{0}=0.0089(2) \AA^{-1}, A=35(20)$, and $B=$ $342(24) \mathrm{K}$. In the second order phase transition, one can fit $\kappa(T)$ above the transition temperature to a power law, $\kappa \sim\left(T-T_{c}\right)^{\nu}$. Due to the limited number of data points in this regime, our fitting did not yield reliable critical exponent $\nu$. To compare with Eq. (1), a power law fit with $\nu=1$ is shown as a dashed line in Fig. 8(b). Note that the power law fit seems to describe the data better in the intermediate temperature range.

In addition, the out-of-plane widths $\kappa_{L}$ broadens as well, which is not as noticeable due to the already quite broad L-width. In order to show this and compare with the in-plane width, $\kappa_{L}$ is divided by 8 , and plotted as diamonds in Fig. 8(b). The out-of-plane width seems to track the temperature dependence of the in-plane widths, which suggests that the CDW correlation becomes more two-dimensional as temperature is raised above $\sim 42 \mathrm{~K}$. A $2 \mathrm{D}$ correlation confined in the ab-plane will manifests itself as a rod of intensity along the L-direction in recipro- cal space, which can be probed in scattering experiments. In Fig. 9(c), the temperature dependence of the L-scans is shown. In order to follow the temperature dependence of the weak and broad peak, we plot the background subtracted intensity. There exists a well-defined peak centered at $\mathrm{L}=5.5$ at $\mathrm{T}=40.3 \mathrm{~K}$, but this peak soon falls to the level of background, while the background intensity increases. As a result, the intensity variation along the L-direction completely vanishes above $\sim 47 \mathrm{~K}$, as shown for $T=47.4 \mathrm{~K}$ and $T=49.4 \mathrm{~K}$, which means that the CDW correlation at these temperatures is completely $2 \mathrm{D}$. This type of 2D-3D crossover can be demonstrated by measuring the temperature dependence of the background point $(\mathrm{L}=5)$, as shown in Fig. 9 (d). Below $\sim 42$ $\mathrm{K}$, the intensity at this point more or less follows the peak intensity, since the out-of-plane correlation is quite short ranged. However, above $\sim 42 \mathrm{~K}$, this intensity grows as temperature is increased, saturating above $\sim 47 \mathrm{~K}$. This observation seems to indicate that dimensional crossover occurs in this temperature range between $42 \mathrm{~K}$ and $47 \mathrm{~K}$.

In Fig. 8 (c), incommensurability (the peak position) as a function of temperature is plotted. The incommensurability changes very little, except for the data point at the highest temperature. Such change of the incommensurability near the CDW transition is not very uncommon, and is believed to arise from the short-ranged nature of the ordering. However, in this case, the change is very small $(\sim 10 \%)$, and does not seem to be directly correlated with the correlation lengths.

\section{Low temperature behavior}

Switching our focus to the low temperature range below $40 \mathrm{~K}$, the peak intensity increases with decreasing temperature and saturates around $15 \mathrm{~K}$, and actually drops below this temperature. The correlation length, on the other hand, does not change below $\sim 40 \mathrm{~K}$. Slight anisotropy in the apparent width along the $\mathrm{H}$ and $\mathrm{K}$ direction is due to the instrumental resolution. Since we observe the same anisotropy $\left(\kappa_{H}>\kappa_{K}\right)$ in both the $(3.23$, $1,5.5)$ peak and the $(3,0.77,5.5)$ peak, we conclude that this is caused by the resolution effect, not by intrinsic anisotropic correlation of stripes.

As in the case of the high temperature change, the peak intensity drops below $15 \mathrm{~K}$ is largely accounted for by the change in the correlation length, since the integrated intensity remains more or less constant. Unfortunately, due to the limitation of the cryostat, our measurements were limited to temperatures over $8 \mathrm{~K}$. However, we have repeated the measurements for different CDW peak positions such as $(4.23,0,5.5)$, and also using the $\mathrm{Si}$ analyzer, which gives instrumental $\mathrm{H}$ and $\mathrm{K}$ resolution of $0.0009 \AA^{-1}$ and $0.0016 \AA^{-1}$, respectively. In all our measurements, the observed behavior could be described consistently by decreasing CDW correlation length below about $15 \mathrm{~K}$. 


\section{DISCUSSION}

According to the temperature dependence study presented in this work, the following physical picture emerges. Above $55 \mathrm{~K}$, our LBCO sample is in LTO structure, and no significant charge order signature is detected. As soon as the LTT structure sets in below 55 $\mathrm{K}$, short range $\mathrm{CDW}$ ordered region appears, since the specific tilting arrangements of the copper oxygen octahedra help stabilizing the CDW order. These patches of CDW has the same structure as the fully developed CDW order, extending up to 4 in-plane lattice units, but there is almost no correlation between next-nearest-neighbor $\mathrm{CuO}_{2}$ layers. However, given that the in-plane correlation lengths are isotropic, neighboring layers should satisfy the crisscrossing pattern. In this sense, this sort of a building block of stripe order might look like the stripe glass phase recently reported in Ref. 43. However, this high temperature short-range stripe correlation in LBCO is probably highly fluctuating. In recent in-plane optical conductivity studies, a decrease in low energy spectral weight was observed below $60 \mathrm{~K}: 27$ Our observation of the dimensional crossover in CDW correlation seems to imply that the c-axis optical conductivity may exhibit different temperature dependence from that of the inplane optical conductivity.

As temperature is cooled down further, the correlation lengths in all three directions expand. In particular, in the temperature range $T=42 \sim 47 \mathrm{~K}$, the $2 \mathrm{D} \mathrm{CDW}$ correlation crosses over to 3D CDW order, although the correlation length along the third direction is very short. The temperature dependence of the correlation length in this temperature regime can be described with phenomenological exponential function or a power law. In the latter case, the critical exponent $0.6 \gtrsim \nu \gtrsim 1.5$ is consistent with our results. Obviously, current data set is too crude to address the universality class of the phase transition, and more accurate measurements are required. However, it is interesting to note that within error bars, the correlation length behavior is consistent with random-field Ising model $(\nu \approx 1.5)$. We note that in recent theoretical studies of charge ordering on a square lattice, the effect of quenched disorder and the resulting random field was considered ${ }^{\underline{44}}$ It is interesting to point out that the short correlation length at high temperature of $\sim 16 \AA$ roughly corresponds to the average distance between Ba dopants in a La-O layer.

Below about $40 \mathrm{~K}$, both the peak intensity and the correlation length grow as temperature decreases until they reach maxima around $15 \mathrm{~K}$. When the sample is cooled further below $15 \mathrm{~K}$, the in-plane correlation length seems to shrink. In our study of the charge stripes in applied magnetic field, $\stackrel{30}{=}$ we observed that the correlation length expands as field is increased above $\sim 5 \mathrm{~T}$, indicating that stripe correlation is enhanced as superconductivity (or fluctuation) is suppressed by either applied field or elevated temperature. We would like to point out the recent transport studies by Li et al.,$\stackrel{29}{,}$ in which they ob- served anomalous drop in in-plane resistivity around 15 $\mathrm{K}$. It was argued that two-dimensional superconductivity sets in at this temperature. Although the relationship between the proposed 2D superconductivity and suppressed CDW correlation is not clear at the moment, it is interesting to note that the important temperature $(15 \mathrm{~K})$ and field $(5 \mathrm{~T})$ scale in both studies match.

In view of recent experimental results, $T \approx 40 \mathrm{~K}$ (perhaps $40-45 \mathrm{~K}$ range) appears to be an important temperature scale. Of course, this temperature is close to the spin density wave ordering temperature $\underline{\underline{39}}$ In addition, $\mathrm{Li}$ et al. observed anomalous jump in thermopower, resistivity ratio, and magnetic susceptibility around $40 \mathrm{~K} \stackrel{29}{2}$ We add to these our observation that the CDW correlation crosses over from $3 \mathrm{D}$ to $2 \mathrm{D}$, and the CDW correlation length is saturated below this temperature. This may suggest that the CDW transition is closely associated with the SDW transition. In LNSCO, it was observed that the CDW order sets in at higher temperature than the SDW order, and this observation was used as evidence that the CDW order drives the SDW order. In LBCO, on the other hand, it was observed that the CDW and SDW seems to order at virtually the same temperature $\stackrel{39}{=}$ Our result suggests that although CDW correlation sets in at higher temperature, both CDW and SDW develop into a relatively long ranged order at the same temperature.

One has to be careful when discussing the CDW and SDW ordering temperatures, since different experimental studies have reported different transition temperatures of charge ordering in LBCO. In their neutron scattering study, Fujita and coworkers reported that the CDW transition and the SDW transition occur at the same temperature $(\sim 45 \mathrm{~K}) \stackrel{39}{=}$ Although the soft x-ray resonant scattering results show CDW intensity up to $55 \mathrm{~K}$, there seems to be an indication that the intensity drops around $45 \mathrm{~K}$. We have measured the temperature dependence utilizing different instrumental resolution, and observed that the apparent transition temperature seems to depend on the momentum resolution. Of course, different samples and different thermometry used in these studies mean that quantitative comparison is not possible. However, the overall trend seems to be that the instrumental resolution of the probing technique does have an effect on the apparent CDW transition temperature.

One of the important questions arising in the discussion of the stripe model is whether the charge stripes are site-centered or bond-centered. This question cannot be answered in our experiments, since we observe incommensurate CDW order. However, if the incommensurate superlattice peaks arise due to discommensuration, the question regarding bond- and site-center remains valid. In this discommensuration model, one assumes that commensurate charge order (in this case period of $\lambda_{0}=4$ ) is separated by regularly spaced domain walls with spacing $d$. If there is a phase slip of $\phi$ at each domain wall, CDW superlattice peaks do not occur at the position corresponding to the inverse of the charge order period: 
$\epsilon=1 / 4$. Instead, they are displaced from the expected superlattice position by the inverse of the large periodicity including the domain walls: $\epsilon=1 / 4+\phi / 2 \pi d$. In our case, this would mean that there exists a regular array of domain walls located at about every 12 atoms, or every three stripes, if we assume $\phi=-\pi / 2$. Therefore, such ordered discommensuration model seems to suggest large number of domain walls in the CDW ordered region, which may be energetically unfavorable. Another test of the discommensuration model is the higher harmonics due to ordered domain walls. Our experimental results showing that there is no significant higher harmonic intensity does not support the discommensuration model. Therefore, the CDW order in our sample seems to be truly incommensurate, which is consistent with SDW ordering in other 214 materials as discussed in Ref. 40.

\section{SUMMARY AND CONCLUSIONS}

We have carried out comprehensive x-ray scattering study of charge stripe ordering in LBCO. We were able to find the following:

1) We found that the first order structural phase transition from the so-called LTO phase to the LTT phase occur at $55 \mathrm{~K}$. In this sample, the LTT phase attains long range order $(\xi>1400 \AA)$ at low temperatures.

2) The LTT phase stabilizes stripes along the direction perpendicular to the tilting of the $\mathrm{CuO}_{6}$ octahedra. We observe only four satellites around each Bragg peak, which shows that the charge density wave (CDW) modulation is only compatible with the one-dimensional stripes.
3) The CDW ground state is described with onedimensional sinusoidal modulation with incommensurate wavevector of $(0.23,0,0.5)$. Any higher harmonics were below our detection limit, which further supports the incommensurate sinusoidal density wave picture.

4) We observed that the CDW correlation is isotropic in the $\mathrm{CuO}_{2}$ plane. That is, the correlation lengths along the crystallographic a- and b-direction remains the same at all temperatures. At low temperatures, these are about 200-250 $\AA$.

5) The correlation length begins to contract above 40 $\mathrm{K}$ in all three directions. In particular, between $42 \mathrm{~K}$ and $47 \mathrm{~K}$, the $3 \mathrm{D}$ to $2 \mathrm{D}$ crossover occurs for the CDW correlation. However, short range isotropic CDW correlation persists up to the LTTLTO phase transition temperature.

6) We were able to explain the L-dependent intensity modulation of the superlattice peaks by considering displacements of La atoms along the c-direction.

\section{Acknowledgments}

We would like to thank J. C. Davis, J. P. Hill, M. Hucker, E. A. Kim, J. H. Kim, S. Kivelson, M. Lawler, Y. S. Lee, A. Paramekanti, S. Sachdev, and J. Tranquada for invaluable discussions. The work at University of Toronto was supported by Natural Sciences and Engineering Research Council of Canada. The work at Brookhaven was supported by the U. S. DOE, Office of Science Contract No. DE-AC02-98CH10886. Use of the Advanced Photon Source was supported by the U. S. DOE, Office of Science, Office of Basic Energy Sciences, under Contract No. W-31-109-ENG-38.
* Electronic address: yjkim@physics.utoronto.ca

1 J. G. Bednorz and K. A. Müller, Z. Phys. B 64, 188 (1986).

2 D. A. Bonn, Nature Physics 2, 159 (2006).

3 M. R. Norman and C. Pepin, Rep. Prog. Phys. 66, 1547 (2003).

4 P. A. Lee, N. Nagaosa, and X. G. Wen, Rev. Mod. Phys. 78, 17 (2006).

${ }^{5}$ P. W. Anderson, P. A. Lee, M. Randeria, T. M. Rice, N. Trivedi, and F. C. Zhang, 16, R755 (2004).

${ }^{6}$ H. Yoshizawa, S. Mitsuda, H. Kitazawa, and K. Katsumata, J. Phys. Soc. Japan 57, 3686 (1988).

7 T. R. Thurston, R. J. Birgeneau, M. A. Kastner, N. W. Preyer, G. Shirane, Y. Fujii, K. Yamada, Y. Endoh, K. Kakurai, M. Matsuda, et al., Phys. Rev. B 40, 4585 (1989).

8 S. W. Cheong, G. Aeppli, T. E. Mason, H. Mook, S. M. Hayden, P. C. Canfield, Z. Fisk, K. N. Clausen, and J. L. Martinez, Phys. Rev. Lett. 67, 1791 (1991).

9 H. Schulz, J. Phys. (Paris) 50, 2833 (1989).

10 D. Poilblanc and T. M. Rice, Phys. Rev. B 39, 9749 (1989).
11 J. Zaanen and O. Gunnarsson, Phys. Rev. B 40, 7391 (1989).

12 V. J. Emery and S. A. Kivelson, Physica C 66, 763 (1994).

13 S. A. Kivelson and V. J. Emery, Strongly Correlated Electronic Materials (Addison-Wesley, Reading, Massachusetts, 1994), pp. 619-656.

14 J. M. Tranquada, B. J. Sternlieb, J. D. Axe, Y. Nakamura, and S. Uchida, Nature 375, 561 (1995).

15 H. Kimura, K. Hirota, H. Matsushita, K. Yamada, Y. Endoh, S.-H. Lee, C. F. Majkrzak, R. Erwin, G. Shirane, M. Greven, et al., Phys. Rev. B 59, 6517 (1999).

16 S. Wakimoto, R. J. Birgeneau, Y. Endoh, P. M. Gehring, K. Hirota, M. A. Kastner, S. H. Lee, Y. S. Lee, G. Shirane, S. Ueki, et al., Phys. Rev. B 60, R769 (1999).

17 Y. S. Lee, R. J. Birgeneau, M. A. Kastner, Y. Endoh, S. Wakimoto, K. Yamada, R. W. Erwin, S. H. Lee, and G. Shirane, Phys. Rev. B 60, 3643 (1999).

18 P. Dai, H. A. Mook, and F. Doğan, Phys. Rev. Lett. 80, 1738 (1998).

19 S. H. Pan, J. P. O'Neal, R. L. Badzey, C. Chamon, H. Ding, 
J. R. Engelbrecht, Z. Wang, H. Eisaki, S. Uchida, A. K. Gupta, et al., Nature 413, 282 (2001).

20 J. E. Hoffman, K. McElroy, D. H. Lee, K. M. Lang, H. Eisaki, S. Uchida, and J. C. Davis, Science 295, 466 (2002).

21 J. E. Hoffman, K. McElroy, D. H. Lee, K. M. Lang, H. Eisaki, S. Uchida, and J. C. Davis, Science 297, 1148 (2002).

22 T. Hanaguri, C. Lupien, Y. Kohsaka, D. H. Lee, M. Azuma, M. Takano, H. Takagi, and J. C. Davis, Nature 430, 1001 (2004).

${ }^{23}$ K. Yamada, C. H. Lee, K. Kurahashi, J. Wada, S. Wakimoto, S. Ueki, H. Kimura, Y. Endoh, S. Hosoya, G. Shirane, et al., Phys. Rev. B 57, 6165 (1998).

24 J. M. Tranquada, H. Woo, T. G. Perring, H. Goka, G. D. Gu, G. Xu, M. Fujita, and K. Yamada, Nature 375, 561 (2004).

25 P. Abbamonte, A. Rusydi, S. Smadici, G. D. Gu, G. A. Sawatzky, and D. L. Feng, Nature Phys. 1, 155 (2005).

26 D. Reznik, L. Pintschovius, M. Ito, S. Iikubo, M. Sato, H. Goka, M. Fujita, K. Yamada, G. D. Gu, and J. M. Tranquada, Nature 440, 1170 (2006).

27 C. C. Homes, S. V. Dordevic, G. D. Gu, Q. Li, T. Valla, and J. M. Tranquada, Phys. Rev. Lett. 96, 257002 (2006).

28 T. Valla, A. V. Fedorov, J. Lee, J. C. Davis, and G. D. Gu, Science 314, 1914 (2006).

${ }^{29}$ Q. Li, M. Hücker, G. D. Gu, A. M. Tsvelik, and J. M. Tranquada, Phys. Rev. Let. 99, 067001 (2007).

30 J. Kim, A. Kagedan, G. D. Gu, C. S. Nelson, T. Gog, D. Casa, and Y. J. Kim, arXiv:cond-mat/0703265.

31 M. v. Zimmermann, A. Vigliante, T. Niemöller, N. Ichikawa, T. Frello, J. Madsen, P. Wochner, S. Uchida, N. H. Andersen, J. M. Tranquada, et al., Europhys. Lett. 41, 629 (1998).
32 T. Niemöller, N. Ichikawa, T. Frello, H. Hünnefeld, N. H. Andersen, S. Uchida, J. R. Schneider, and J. M. Tranquada, 12, 509 (1999).

33 S. Wakimoto, R. J. Birgeneau, Y. Fujimaki, N. Ichikawa, T. Kasuga, Y. J. Kim, K. M. Kojima, S.-H. Lee, H. Niko, J. M. Tranquada, et al., Phys. Rev. B 67, 184419 (2003).

34 H. Kimura, H. Goka, M. Fujita, Y. Noda, K. Yamada, and N. Ikeda, Phys. Rev. B 67, 140503 (2003).

${ }^{35}$ R. J. Birgeneau, C. Y. Chen, D. R. Gabbe, H. P. Jenssen, M. A. Kastner, C. J. Peters, P. J. Picone, T. Thio, T. R. Thurston, H. L. Tuller, et al., Phys. Rev. Lett. 59, 1329 (1987).

36 J. D. Axe, A. H. Moudden, D. Hohlwein, D. E. Cox, K. M. Mohanty, A. R. Moodenbaugh, and Y. Xu, Phys. Rev. Lett. 62, 2751 (1989).

37 S. Katano, J. A. Fernandez-Baca, S. Funahashi, N. Mori, Y. Ueda, and K. Koga, Physica C 214, 64 (1993).

38 Y. Zhu, A. R. Moodenbaugh, Z. X. Cai, J. Tafto, M. Suenaga, and D. O. Welch, Phys. Rev. Lett. 73, 3026 (1994).

39 M. Fujita, H. Goka, K. Yamada, J. M. Tranquada, and L. P. Regnault, Phys. Rev. B 70, 104517 (2004).

40 J. Robertson, S. A. Kivelson, E. Fradkin, A. Fang, and A. Kapitulnik, Phys. Rev. B 74, 134507 (2006).

41 J. M. Tranquada, J. E. Lorenzo, D. J. Buttrey, and V. Sachan, Phys. Rev. B 52, 3581 (1995).

42 J. M. Tranquada, N. Ichikawa, and S. Uchida, Phys. Rev. B 99, 14712 (1999).

${ }^{43}$ Y. Kohsaka, C. Taylor, K. Fujita, A. Schmidt, C. Lupien, T. Hanaguri, M. Azuma, M. Takano, H. Eisaki, H. Takagi, et al., Science 315, 1380 (2007).

44 A. Del Maestro, B. Rosenow, and S. Sachdev, Phys. Rev. B 74, 024520 (2006). 